\title{
Bleomycin mouse model with active-phase pulmonary fibrosis and no acute mortality
}

\author{
ANIL H KADAM \\ Proteogenomic Research Institute for Systems Medicine \\ Peggy H. Faix ( $\nabla$ pfaix@prism-sd.org ) \\ Jan E. Schnitzer \\ PRISM
}

\section{Research}

Keywords: bleomycin, pulmonary fibrosis, disease biomarkers, lung disease, inflammation

Posted Date: February 11th, 2020

DOI: https://doi.org/10.21203/rs.2.23139/v1

License: (9) This work is licensed under a Creative Commons Attribution 4.0 International License. Read Full License 


\section{Abstract}

Background The bleomycin mouse model is the most prevalent animal model used to study idiopathic pulmonary fibrosis (IPF), ranging from basic pathophysiological mechanisms to therapeutic modalities. High mortality rates, inconsistencies in disease induction, and great severity of the disease phenotype have limited its utility and clinical relevance. In this study, we identify conditions in the mouse bleomycin model that maintain the desired IPF phenotype found in patients while avoiding the usual, associated rapid mouse mortality.

Methods In two independent studies, we analyzed the effects of incremental intratracheal (IT) high (1-3 $\mathrm{U} / \mathrm{kg}$ ) and low (0.05-0.5 U/ kg) bleomycin doses on pulmonary fibrosis in mice. We observed dose effects on weight loss, morbidity and mortality, inflammation, lung collagen content, presence of various biomarkers in bronchoalveolar lavage fluid (BALf), and histology 14 days post-treatment, when the animals were in the active phase of fibrosis.

Results We characterize the fibrotic effects of various bleomycin doses in mice on pulmonary inflammation, myofibroblast activation, vascular leakiness, angiogenesis, and extracellular tissue remodeling. Higher bleomycin doses induced acute disease with severe and saturated responses, extreme and progressive weight loss, and high morbidity and mortality rates. In contrast, lower doses of bleomycin induced a milder and chronic response, featuring robust, active-phase fibrosis with mild weight loss and no mortality.

Conclusion Here we demonstrate that the bleomycin mouse model can be used to reproduce symptoms of IPF patients in an animal by using low doses of bleomycin. Low concentrations of bleomycin induce chronic and progressive fibrosis with no mortality.

\section{Background}

Idiopathic pulmonary fibrosis (IPF) is a devastating, progressive disease marked by excessive scarring, which leads to increased tissue stiffness, loss in lung function, and, ultimately, death. Recent studies suggest an increasing prevalence of IPF and growing numbers of associated mortality [1]. IPF is characterized by progressive fibroblast and myofibroblast proliferation and extensive deposition of extracellular matrix (ECM) [2], resulting in abnormal remodeling of the lung parenchyma. The determination of disease mechanisms remains a difficult challenge in the field, and currently there are no reliably effective therapies for treating IPF in the clinic.

Animal models are essential for defining basic pathophysiological mechanisms and identifying new therapeutic agents. Models that closely simulate the symptoms of disease in patients are essential to improve clinical success rates for drug testing [3]. The bleomycin mouse model is the most prevalent system used for studying IPF. Single bleomycin administration effectively replicates several of the pathogenic molecular changes associated with IPF and is used as a model for patients with active disease [4]. 
While this model has proven useful in examining may aspects of IPF, such as understanding the pathology of disease, identifying potential targets for medication, and establishing all treatments currently in use [5], high mortality and extreme disease severity limit its clinical utility. High doses of bleomycin induce intense and acute fibrosis and high rates of mortality in animals, whereas in patients, IPF manifests as a slow, chronic decline in health spanning several years [1]. In past studies utilizing the bleomycin mouse model, inconsistent rates of mortality and disease magnitude are reported over the course of a few weeks [6-8]. Death and intense disease severity mask the pathobiological effects that are essential to understanding disease mechanism and pathology and limit this model's clinical utility.

We report here the relationship between bleomycin dose and fibrotic endpoints with the objective of improving the bleomycin mouse model by inducing a milder, less acute disease that avoids mortality. To address the shortcomings of the model's current utilization, we report the effect of a wide range of bleomycin doses on pulmonary inflammation, vascular leakiness, and remodeling of lung architecture. These data provide the conditions of the bleomycin mouse model that produce a chronic disease phenotype that mirrors the clinical symptoms of IPF, affording a more clinically relevant disease model.

\section{Material And Methods}

\section{Animals}

Experiments were carried out with the approval of the PRISM Institutional Animal Care and Use Committee. 10- to 12-weeks-old C57BL/6J female mice (Jackson Laboratory, USA) were used for the studies. Animals were acclimatized for a period of 7 days in pathogen-free conditions, under a $12 \mathrm{~h}$ normal-phase, light-dark cycle with food and water ad libitum. Animals were maintained under these conditions until the end of the study.

\section{Induction of pulmonary fibrosis and times for endpoint analysis}

To induce pulmonary fibrosis, we introduced pharmaceutical-grade bleomycin (Zydus-Hospira Oncology Private Ltd., India) dissolved in 1X PBS by intratracheal (IT) injection. To observe dose-dependent effects of bleomycin on various endpoints of disease phenotype in mice, we executed two independent experiments. For the high-dose response study, groups of randomly divided mice $(n=5)$ were dosed IT with $1 \mathrm{U} / \mathrm{kg}, 1.5 \mathrm{U} / \mathrm{kg}, 2 \mathrm{U} / \mathrm{kg}$, or $3 \mathrm{U} / \mathrm{kg}$ bleomycin, or PBS only. Groups of mice in the low-dose response study ( $\mathrm{n}=5)$ were treated IT with $0.05 \mathrm{U} / \mathrm{kg}, 0.1 \mathrm{U} / \mathrm{kg}, 0.25 \mathrm{U} / \mathrm{kg}$, or $0.5 \mathrm{U} / \mathrm{kg}$ bleomycin, or PBS only. We began with high-dose response studies and executed the low-dose experiments based on observations and outcomes of the former. Briefly, on day 0, female C57BL/6J (8-10 weeks) mice were anesthetized by intraperitoneal injection of Xylazine: ketamine $(10: 80 \mathrm{mg} / \mathrm{kg})$ and then challenged with a single IT injection of bleomycin dissolved in $50 \mu \mathrm{L}$ of PBS with a 31-gauge needle attached to a Hamilton syringe. Control mice received $50 \mu \mathrm{L}$ of PBS only. Animals were sacrificed on day 14 post- challenge. Animals 
were euthanized with Euthasol (100-120 mg/kg), and bronchoalveolar lavage (BAL) was performed. The lungs were collected for estimation of lung collagen content and histopathology.

\section{BAL and sample collection}

Animals were sacrificed, and BAL was performed. Briefly, mice were terminally anaesthetized, and the trachea was cannulated using a 20-gauge catheter. BAL was performed 4 times using $0.3 \mathrm{ml}$ of $1 \mathrm{X}$ PBS ( $\mathrm{pH}$ 7.4). The total volume of each BAL aspirate was pooled. A $20 \mu \mathrm{L}$ aliquot of BAL fluid (BALf) was mixed with $20 \mu \mathrm{L}$ of Turk's solution for the leukocytes counts. Aliquots of $120 \mu \mathrm{L}$ BALf were collected, and the remaining fluid was centrifuged at 10,000 RPM for 10 min at $4^{\circ} \mathrm{C}$. $250 \mu \mathrm{L}$ aliquots of centrifuged BALf were collected and stored at $-80^{\circ} \mathrm{C}$ for cytokine estimations. Cell pellets were mixed with rat serum to prepare smears on frosted glass slides. After BAL, the right lungs were harvested, washed in 1X PBS, and stored at $-80^{\circ} \mathrm{C}$ for lung collagen estimation. The left lungs were collected and fixed in $10 \%$ neutral formalin buffer (NBF) for histology.

\section{Assessment of general health and pulmonary inflammation}

All mice were monitored daily for health, weight loss, and mortality over the entire duration of the study. To assess pulmonary inflammation, BALf samples were analyzed for total leukocytes and differential cells counts. Total leukocyte count was performed by mixing BALf 1:1 with Turk's solution, and cells were counted using a hemocytometer (Hausser Scientific Horsham, PA, USA). Total differential cell counts were performed at 100X magnification using light microscopy (BX2, Olympus, Tokyo, Japan). 500 cells were stained with Leishman stain and cells manually evaluated by morphology and counted macrophages, lymphocytes, or neutrophils.

\section{Assessment of right lung collagen content, lung weight, and lung index}

Fibrosis was assessed by quantifying total soluble collagen in the lung using the Sircol collagen assay kit (Biocolor Ltd., UK). Briefly, wet right lungs were weighed and homogenized in $2 \mathrm{~mL}$ of CHAPS buffer. The lung homogenate was mixed with an equal volume of acid pepsin solution $(5 \mathrm{mg} / \mathrm{ml}$ of $0.5 \mathrm{M}$ acetic acid), incubated overnight at $4^{\circ} \mathrm{C}$, centrifuged, and the supernatant was assayed for soluble collagen according to the manufacturer's instructions. Absorbance at $555 \mathrm{~nm}$ was read on a VersaMax ELISA microplate reader (Molecular Devices, LLC. CA, USA). Lung collagen data were expressed as $\mu \mathrm{g}$ of soluble collagen per right lung. To obtain the lung index (i.e. lung weight to weight ratio), the wet weight of right lung (mg) was divided by weight $(\mathrm{g})$ of animals.

\section{Assessment of profibrotic environment of lung and vascular leakiness.}

Profibrotic biomarkers of lung fibrosis were analyzed by assessing cytokine levels in BALf, determined by using commercially available ELISA assays, following to the manufacturer's instructions. Quantikine ELISA kit for TGF- $\beta 1$, IL-6, TIMP1, VEGF, IL-13, C-reactive protein (CRP), and WISP1 (R\&D Systems, San 
Diego, CA, USA). Colorimetric detection of protein concentration in BALf supernatants was measured by using the bicinchoninic acid (BCA) assay. Absorbances were determined at $570 \mathrm{~nm}$ using a spectrophotometer, and proteins concentrations were calculated based on a bovine serum albumin (BSA) standard curve.

\section{Assessment of histopathology and severity of lung fibrosis}

Animals were sacrificed. Left lungs were removed and stored in 10\% NBF. The lung tissues were processed per routine histopathology procedure. Briefly, lung tissue was embedded in paraffin and then cut into $5 \mu \mathrm{m}$ thick sections that were then stained with hematoxylin and eosin (H\&E). The severity of interstitial fibrosis among the groups was compared per the modified Ashcroft scale, as described by Ashcroft and co-workers [9].

\section{Statistical Analysis}

All data are presented as the mean \pm standard error of the mean (S.E.M.). The data were analyzed with one-way ANOVA, followed by Dunnett's test for multiple comparisons, comparing to controls. A $p$-value < 0.05 was set as statistically significant.

\section{Results}

\section{High-dose bleomycin induction of pulmonary fibrosis}

Bleomycin induces severe weight loss and mortality.

We assessed the dose-response effect of 1-3 $\mathrm{U} / \mathrm{kg}$ bleomycin administered via IT injection on clinical signs such as weight loss, mortality, and health, examined daily. We observed significant $(p<0.001)$ dosedependent mortality in bleomycin-challenged mice compared to control, PBS-treated animals. The percent survival was $100 \%, 83 \%, 44 \%$, and $0 \%$, corresponding to $1,1.5,2$, and $3 \mathrm{U} / \mathrm{kg}$ bleomycin challenge, respectively (Fig. 1a). Animals treated at these doses also exhibited significant weight loss compared to controls. The percentage of weight change was progressive and severe from day 0 of bleomycin challenge to the endpoint. The mice did not recover from weight loss (Fig. 1b). An average of $15-25 \%$ weight loss was observed at $1-2 \mathrm{U} / \mathrm{kg}$ bleomycin, and challenge with $3 \mathrm{U} / \mathrm{kg}$ bleomycin resulted in $45 \%$ loss (Fig. 1C).

Bleomycin induces severe pulmonary inflammation.

We assessed the inflammatory response in animals challenged with IT administration of $1,1.5$, and 2 $\mathrm{U} / \mathrm{kg}$ bleomycin. As noted previously, animals did not survive bleomycin treatment at $3 \mathrm{U} / \mathrm{kg}$. BALf samples were harvested, and the inflammatory response was assessed by performing differential cell count. We observed an 8- to 14-fold increase $(p<0.001)$ in total leucocytes count (Fig. 1d). Differential counts indicate significant increases in levels of macrophages $(p<0.001)$, lymphocytes $(p<0.01)$, and neutrophils $(p<0.05)$ in bleomycin-challenged animals. The pulmonary inflammation observed in treated 
animals was due to infiltration of lymphocytes (45-60\%), macrophages (35-45\%), and neutrophils (4$12 \%$ ) in response to injury from bleomycin challenge, compared to controls (Fig. 1e-g, respectively).

Bleomycin induces severe lung fibrosis and increases vascular leakiness.

We assessed the dose effects of bleomycin challenge on lung parameters, including measurement of lung collagen content, lung weight, and lung index. Animals treated with $1,1.5$, and $2 \mathrm{U} / \mathrm{kg}$ showed a 9- to 11 -fold significant increase in lung collagen content $(p<0.001)$, a 2.1 - to 2.5 -fold increase in lung weight $(p<0.001)$, and a 1.7- to 1.9-fold increase in lung index ( $p<0.001)$, respectively, compared to controls (Fig. 2a-c, respectively). Vascular leakiness was analyzed by estimating protein content in BALf samples. We observed a significant increase in BALf protein content $(p<0.001)$, averaging 19 - to 27 -fold higher with bleomycin treatment than controls (Fig. 3a).

Bleomycin increases levels of BALf biomarkers and fibrosis mediators.

We tested the effect of bleomycin dose escalation on biomarkers that play a key role in pulmonary fibrosis. BALf samples were collected following treatment with increasing doses of bleomycin (1-2 $\mathrm{U} / \mathrm{kg}$ ). We examined the effect of profibrotic mediators involved in the activation of myofibroblasts in response to bleomycin challenge. Results showed a significant increase in levels of TGF- $\beta 1$ and VEGF ( $p$ $<0.001$ ) (Fig. 3b and d, respectively), compared to controls, whereas we observed a highly variable, insignificant increase of IL-6 (Fig. 3c). Bleomycin induced a marked increase in TIMP1, a biomarker of tissue remodeling in pulmonary fibrosis, with levels significantly increased $(p<0.001)$ over control animals (Fig. 3e). Similarly, the levels of CRP, a biomarker indicative of decline in lung function, were significantly higher $(p<0.001)$ in treated mice compared to controls (Fig. $3 f)$. These results indicate that the level of relevant biomarkers of pulmonary fibrosis are all significantly elevated in bleomycin-treated compared to control animals; however, little difference was discerned between these doses with the methods of detection utilized here.

\section{Low-dose bleomycin induction of pulmonary fibrosis}

Bleomycin induces dose-dependent weight loss without mortality.

In addition to the high mortality and disease severity in mice in response to high doses $(>1 \mathrm{U} / \mathrm{kg}$ ) of bleomycin, we established a dose-response relationship using lower doses $(0.05-0.5 \mathrm{U} / \mathrm{kg})$. A single IT challenge with bleomycin at $0.1,0.25$, or $0.5 \mathrm{U} / \mathrm{kg}$ induced significant weight loss $(p<0.05)$, but this was not evident at $0.05 \mathrm{U} / \mathrm{kg}$ bleomycin, as compared to control animals (Fig. 4a-b). In the 0.25 and $0.5 \mathrm{U} / \mathrm{kg}$ bleomycin treatment groups, weight loss was progressive until day 7 , at which point it stabilized between days $7-10$, followed by weight recovery until sacrifice. In contrast, animals challenged with 0.05 and 0.1 $\mathrm{U} / \mathrm{kg}$ bleomycin did not exhibit significant weight loss throughout the duration of the 14-day study (Fig. 4a). Animals averaged 1.4, 4.84, 7.32, and $8.46 \%$ weight loss in a dose-dependent manner in response to challenges with $0.05,0.1,0.25$, or $0.5 \mathrm{U} / \mathrm{kg}$ bleomycin, respectively (Fig. $4 \mathrm{~b}$ ). No mortality was observed in any of the low-dose bleomycin-challenged groups. 
Bleomycin induces dose-dependent pulmonary inflammation.

We analyzed BALf samples to assess the dose-dependent response of $0.05-0.5 \mathrm{U} / \mathrm{kg}$ bleomycin on inflammation, as described above, and calculated a 3- to 14-fold increase in total leukocyte count that was markedly significant $(p<0.01)$ at $0.1-0.5 \mathrm{U} / \mathrm{kg}$ bleomycin (Fig. $4 c)$. Macrophage levels significantly increased $(p<0.01)$ in animals treated with $0.1,0.25$, and $0.5 \mathrm{U} / \mathrm{kg}$ bleomycin over controls, and the increase was more modest in animals dosed at $0.05 \mathrm{U} / \mathrm{kg}$ bleomycin (Fig. 4d). The lymphocyte levels also dose-dependently increased and was most significant $(p<0.01)$ between $0.1-0.5 \mathrm{U} / \mathrm{kg}$ bleomycin (Fig. 4e). A similar pattern was observed in neutrophil levels, with significant $(p<0.01)$ increases at 0.25 and $0.5 \mathrm{U} / \mathrm{kg}$ (Fig. 4f). Overall, the increase in total BALf cells can be summarized as $65-85 \%$ macrophages, $12-30 \%$ lymphocytes, $2-4 \%$ neutrophils, and $0 \%$ eosinophils. This is in marked contrast to the cell count of control samples that were solely macrophages (Fig. 4c-f).

Bleomycin induces dose-dependent increases in vascular leakiness and lung fibrosis.

Vascular leakiness in response to low-dose bleomycin challenge was assessed using the same methods utilized in the high-dose studies. BALf samples were obtained from $0.05-0.5 \mathrm{U} / \mathrm{kg}$ bleomycin- and PBSchallenged mice. Dose-dependent, elevated levels of BALf protein content were observed in bleomycintreated mice as compared to controls and found to be most significant $(p<0.01)$ in animals challenged with $0.1-0.5 \mathrm{U} / \mathrm{kg}$ bleomycin. Increased BALf protein content was 3.3-, 4.6-, 8.8-, and 11.3-fold in response to treatment with $0.05,0.1,0.25$, and $0.5 \mathrm{U} / \mathrm{kg}$ bleomycin, respectively, over controls (Fig. $5 \mathrm{~d}$ ). The effect of treatment on lung parameters was also assessed. The administration of $0.1-0.5 \mathrm{U} / \mathrm{kg}$ bleomycin significantly $(p<0.01)$ elevated lung collagen content, lung weight, and lung index in a dosedependent manner, compared to control animals. Increased lung collagen content was 3.2-, 5.3-, and 8.7fold greater than controls in mice treated with $0.1,0.25$, and $0.5 \mathrm{U} / \mathrm{kg}$ bleomycin, respectively (Fig. $5 \mathrm{a}$ ). Lung weight was 1.5, 1.8, and 1.9-fold (Fig. 5b) increased, and lung index was 1.5, 1.8, and 1.9-fold (Fig. 5c) increased in mice challenged with $0.1,0.25,0.5 \mathrm{U} / \mathrm{kg}$ bleomycin, respectively. No significant increase in collagen content (1.5-fold), wet weight of right lung (1.1-fold), nor lung index (1.1-fold) was observed in mice dosed with $0.05 \mathrm{U} / \mathrm{kg}$ bleomycin, compared to controls (Fig. 5a-c).

Bleomycin induces dose-dependent increases of BALf fibrosis biomarkers.

We assessed the effect of $0.05-0.5 \mathrm{U} / \mathrm{kg}$ bleomycin challenge on levels of profibrotic biomarkers, specifically TGF- $\beta 1$, IL-13, IL-6, WISP1, and VEGF, present in BALf samples. TGF $\beta-1$ (Fig. 6a), IL-6 (Fig. 6c), WISP1 (Fig. 6d), and VEGF (Fig. 6e) levels were elevated in a dose-dependent manner, whereas IL-13 levels (Fig. 6b) did not respond accordingly. Significant increases $(p<0.05)$ in levels of TGF $\beta-1$, IL-13, and WISP1 were observed at all doses of bleomycin. In contrast, IL-6 levels significantly increased $(p<0.05)$ at $0.25-0.5 \mathrm{U} / \mathrm{kg}$ bleomycin, while VEGF levels increased significantly $(\mathrm{p}<0.05)$ only at $0.5 \mathrm{U} / \mathrm{kg}$ bleomycin, compared to controls. We also observed dose-dependent responses to bleomycin challenge in the levels of TIMP1 and CRP (Fig. 6f-g, respectively), which are used as biomarkers of lung architecture remodeling and function. In $0.25-0.5 \mathrm{U} / \mathrm{kg}$ bleomycin-challenged mice, increased levels were detected of TGF1- $\beta-1$ (4-6-fold), IL-13 (4-5 fold), IL-6 (8-13 fold), WISP1 (35-42 fold), VEGF (1.2-1.36 fold), TIMP1 (1,500- 
2,500 fold), and CRP (34-39 fold), compared to control. In $0.1 \mathrm{U} / \mathrm{kg}$ bleomycin-challenged mice, elevated levels of TGF- $\beta 1$ (1.8-fold), IL-13 (2.5-fold), IL-6 (2.4-fold), WISP1(13-fold), VEGF (0.8-fold), TIMP1 (164fold), and CRP (20-fold) were observed over control. At $0.05 \mathrm{U} / \mathrm{kg}$ bleomycin challenge, increases in levels of TGF- $\beta 1$ (1.6-fold), IL-13 (3.54-fold), IL-6 (2.42-gold), WISP1 (6.87-fold), VEGF (0.75-fold), TIMP1 (92fold), and CRP (13.2-fold) were observed, over control mice (Fig. 6a-g).

Bleomycin induces dose-dependent fibrotic changes and severity in lung tissue.

In histological examinations of harvested lungs, the control and $0.05 \mathrm{U} / \mathrm{kg}$ bleomycin-treated animals (Fig. 7a-b, respectively) revealed predominately normal lung architecture. However, in animals dosed with $0.1 \mathrm{U} / \mathrm{kg}$ bleomycin, histology revealed profound fibrotic changes exhibited as disjointed, knot-like formations, contiguous fibrotic walls, and fibrotic masses (Fig. 7c). Furthermore, mouse lungs collected from animals treated with 0.25 and $0.5 \mathrm{U} / \mathrm{kg}$ bleomycin (Fig. $7 \mathrm{~d}$-e, respectively) revealed significant infiltration of inflammatory cells, thickening of alveolar septa, damage to the lung tissue, and the deposition of extracellular matrix in lung parenchyma. The fibrotic changes in the lungs were quantified using the modified Ashcroft scale. We found a positive correlation between concentrations of bleomycin and lung architecture loss and fibrosis (Fig. 7f). Consistent with other features of fibrosis that we investigated, dose-dependent changes were detected.

In all, with high concentrations of bleomycin administration, we elicited acute pulmonary fibrosis in mice accompanied by high mortality and progressive, severe weight loss. We also successfully induced fibrosis in mice using $<1 \mathrm{U} / \mathrm{kg}$ bleomycin with no mortality. At these lower concentrations, we established a dose-dependent relationship between bleomycin concentration and disease severity and confirmed this relationship both at the molecular and gross level.

\section{Discussion}

In the present study, we systematically investigated the effects of varying doses of bleomycin on developing pulmonary fibrosis in mice by characterizing a set of endpoints. We elicited a reliable and incremental disease phenotype in response to low-dose $(0.05-0.5 \mathrm{U} / \mathrm{kg})$ bleomycin treatment. In contrast, we observed a severe response and mortality in animals that were treated IT with high $(1-3 \mathrm{U} / \mathrm{kg})$ bleomycin doses. Our data present the calibrated response of mice to bleomycin challenge that will be useful for future research and drug development efforts to understand further the mechanisms of pulmonary fibrosis and its response to novel therapies.

In reading past reports, we found difficulties in comparing various studies. Inconsistencies in the source and quality (research-grade or pharmaceutical-grade) of bleomycin greatly impacts outcomes $[4,10,11]$. Furthermore, doses of bleomycin are reported with differing units $(\mathrm{U} / \mathrm{kg}$ or $\mathrm{mg} / \mathrm{kg}$ ) without traceable, stoichiometric conversions. This brings about limitations in comparing findings across studies and in comparing our data with previous reports. Therefore, despite the plethora of IPF studies that have been conducted, we compare our data with only a handful that, like us, used pharmaceutical-grade bleomycin with doses measured in $\mathrm{U} / \mathrm{kg}$. Common across most studies is administration of high doses of 
bleomycin. Here we show that low doses still induce fibrotic profiles but without acute disease and mortality.

With high doses $(1-3 \mathrm{U} / \mathrm{kg})$ of bleomycin, we observed progressive yet severe weight loss without recovery. Mortality was observed starting as early as 7 days post-bleomycin treatment. In the end, $56 \%$ and $100 \%$ mortality were observed with 2 and $3 \mathrm{U} / \mathrm{kg}$ bleomycin, respectively. This is consistent with previous reports of $40 \%$ and $90 \%$ mortality using 1.5 and $2.5 \mathrm{U} / \mathrm{kg}$ IT bleomycin, respectively [6]. However, other studies report no mortality with $1.5 \mathrm{U} / \mathrm{kg}$ bleomycin administered by oropharyngeal aspiration, suggesting that different methods of drug administration generate distinct responses in this model [1820].

Our endpoint analysis of mice treated with 1-2 U/kg bleomycin showed significantly increased levels of inflammation involving lymphocytes, macrophages, and neutrophils compared to controls. Additionally, we observed statistically significant increases in collagen content in the lung, accompanied by increased lung weight and lung index in treated animals compared to untreated mice. Analysis of biomarkers in BALf showed significant increases in TGF- $\beta 1$, IL-6, VEGF, TIMP1, and CRP levels, all of which are consistent with previous reports [12-17].

Because we observed high mortality and severe weight loss in mice challenged with 1-3 U/kg bleomycin, we reasoned that incrementally dosing lower concentrations of bleomycin $(<1 \mathrm{U} / \mathrm{kg})$ would avoid acute, lethal disease, but we were unsure if lung fibrosis would continue to be induced. Decreasing doses may allow us to capture a more granular understanding of the fibrotic responses that were likely lost or masked in the high-dose studies and allow us to reveal the correct dosage needed to elicit a chronic form of disease.

As we aimed to achieve, mice administered $0.1,0.25$, or $0.5 \mathrm{U} / \mathrm{kg}$ bleomycin IT exhibited significant, dosedependent weight loss without mortality but still clearly exhibited fibrotic profiles. Weight loss capped at $8.46 \%$ in the $0.5 \mathrm{U} / \mathrm{kg}$ bleomycin-treated mice. At 0.25 and $0.5 \mathrm{U} / \mathrm{kg}$ bleomycin, weight declined until day 7 , stabilized until day 10, and then recovered up until the time of sacrifice. Similarly, past reports show that bleomycin induces severe and progressive weight loss up to days 7-10 post-treatment, followed by recovery from day 10 onward until sometimes complete recovery at day 21 [18].

We observed dose-dependent inflammation, characterized by significant 3- to 14-fold increases in total counts of leukocytes, macrophages, lymphocytes, and neutrophils. In total, macrophages dominated the cell counts at 65-85\%, while lymphocytes were comprised $12-30 \%$ and neutrophils made up 2-4\% of total cell count. Counts in control mice were $100 \%$ macrophages.

A similar dose-dependent pattern was observed in lung collagen content, lung weight, and lung index. All three parameters increased with bleomycin concentration. Measurement of protein concentration of BALf revealed vascular leakiness. Increasing concentrations of bleomycin corresponded with increasing BALf protein concentration. These readouts are all consistent with past reports [19]. 
We also examined pathophysiological mechanisms involved in the fibrotic response. Levels of clinically relevant, molecular targets and mediators of fibrosis were analyzed in BALf samples collected from mice subjected to $0.05-0.5 \mathrm{U} / \mathrm{kg}$ bleomycin. Results showed dose-dependent changes in the expression levels of key pro-fibrotic cytokines and growth factors involved in the activation of myofibroblasts during fibrogenesis, mediation of inflammation, angiogenesis, matrix remodeling, and indicators of lung function.

We show increased TGF- $\beta 1$ levels with bleomycin treatment. This is supported by past studies that found that TGF- $\beta 1$ has a pivotal role in the development of tissue fibrosis in IPF [20, 21]. It modulates transcription of downstream target genes, including procollagen I and III, via transmembrane receptor serine/threonine kinases and the cytoplasmic Smad-2/3 signaling pathways [22]. TGF- $\beta 1$ promotes ECM accumulation, specifically collagen and fibronectin, and drives phenotypic changes in fibroblasts [23].

IL-13 content increased to similar levels at all doses of bleomycin, but all were above IL-13 levels in untreated mice. IL-13, a Th2 cytokine is increased in the blood and BALf of patients with IPF, and its levels are directly correlated with disease severity [24]. IL-13 promoted pulmonary fibrosis in radiation-induced lung fibrosis models, and IL-13 inhibition decreased fibrotic changes in pulmonary fibrosis in mice [25]. Mechanistically, IL-13 differentiates human lung fibroblasts to myofibroblasts through a c-Jun N-terminal kinases-dependent pathway [26]. In a recent study utilizing the bleomycin mouse model, sphingosine-1phosphate receptor-2 (S1PR2) was found to facilitate pulmonary fibrosis through potentiating the IL-13 pathway in macrophages [27].

We also observed IL-6 levels increase dose-dependently in mice. IL- 6 is a pleiotropic cytokine and functions as a proinflammatory and profibrotic factor in fibrosis pathogenesis [28]. It is expressed and secreted by various cells, including alveolar macrophages, lung fibroblasts, and fibrocytes [29]. In addition to the TGF- $\beta$ /Smad3 pathway, the signaling loop of IL-6/gp130/Stat3 has a crucial role in disease pathogenesis [30], and blockade of IL-6 signaling during the chronic stages of lung injury has a beneficial effect on lung fibrosis [31].

Similarly, we observed increased levels of WISP1 in all doses of bleomycin. WISP1 mediates IL-6dependent cell proliferation through a mechanism orchestrated by a variety of profibrotic biomarkers, including Wnts, TGF- $\beta 1$, and TNF-a, in primary human lung fibroblasts [32]. In vivo increases of WNT/ $\beta$ catenin signaling leads to the expression of the proinflammatory cytokines IL- $1 \beta$ and IL- 6 in alveolar epithelial type cells in the bleomycin mouse model $[33,34]$. Dysregulated activation of WISP1 plays a key role in IPF [35], and heightened expression of WISP1 in AECII cells was reported in both the mouse bleomycin model and IPF patients. Neutralizing WISP1 with antibodies attenuates lung fibrosis, reduces collagen deposition, expresses genes associated with epithelial-mesenchymal transition, and increases survival in the bleomycin mouse model [36].

We observed elevated levels of VEGF and TIMP1 with bleomycin treatment, indicating angiogenesis, which is a prominent histopathological feature of IPF. This is supported by past reports that implicate these proteins in fibrogenesis. VEGF is a biomarker for angiogenesis and is the target of several IPF 
therapies [37]. TIMP1 is a protein expressed on macrophages and epithelial cells that promotes cell proliferation [38-40]. TIMP1 showed a greater fold increase than the other biomarkers we assessed, suggesting that it may be a primary contributor to fibrogenesis.

We detected a direct, dose-dependent correlation between bleomycin concentration and CRP levels. CRP levels can potentially be included as a predictive biomarker for lung function analysis in the bleomycin mouse model like in clinical studies. In patients CRP is used as biomarker of decline in lung function and a determinant of survival in respiratory illnesses. High CRP levels are predictive of short survival time and greater deterioration of lung activity [41, 42].

Lastly, we used histopathology to confirm our molecular and mechanistic readouts with higher-level, physical changes in lung tissue that resulted from bleomycin treatment. We utilized the modified Ashcroft scale to correlate the severity of fibrosis with bleomycin insult [9]. Lung architecture was preserved in animals treated with $0.05 \mathrm{U} / \mathrm{kg}$ bleomycin and was similar to lungs of controls. $0.1 \mathrm{U} / \mathrm{kg}$ bleomycin induced moderate fibrotic changes, and 0.25 and $0.5 \mathrm{U} / \mathrm{kg}$ elicited profound changes, such as deposition of fibrotic masses in lung tissue. These observations are consistent with results of previous studies that utilized bleomycin in the high-dose range. Although these doses are much lower than those used in some previous studies $[10,15]$, we achieved similar levels of disease severity.

\section{Conclusion}

In summary, we present conditions to achieve chronic fibrosis in the bleomycin model by using $<1 \mathrm{U} / \mathrm{kg}$ bleomycin administered by IT. At these doses, we observed no mortality, mild and recoverable weight loss, and concentration-dependent changes in biomarkers and inflammation. These features are more reminiscent of clinical phenotypes of IPF patients than most past studies, in which high bleomycin doses elicited acute, severe fibrosis with mortality. We hope that our proposed conditions will assist in dissecting the mechanism and pathobiology of IPF and ultimately lead to more promising therapies.

\section{Abbreviations}

IPF

Idiopathic pulmonary fibrosis

IT; IntratrachealTGF- $\beta 1$

Transforming growth factor beta

IL-6

Interleukin-6

IL-13

Interleukin-13

WISP-1

WNT1-inducible-signaling pathway protein 1

VEGF

Page $11 / 21$ 
Vascular endothelial growth factor

TIMP-1

Tissue inhibitor of metalloproteinase

CRP

C-reactive protein

ECM

Extracellular matrix

PRISM

Proteogenomics Research Institute for Systems Medicine

IACUC

Institutional Animal Care and Use Committee

BAL

Bronchoalveolar lavage

BALf

Bronchoalveolar lavage fluid

NBF

Neutral formalin buffer

ELISA

Enzyme-linked immunosorbent assay

BSA

Bovine serum albumin

$H \& E$

Hematoxylin and eosin

SEM

Standard error of the mean

S1PR-2

Sphingosine-1-phosphate receptor-2

\section{Declarations}

Ethics approval and consent to participate

All experimental procedures were approved by PRISM Institutional Animal Care and Use Committee (Reference number 14-01).

Consent for publication

Not applicable 
Availability of data and materials

Source data and material will be available upon request.

\section{Competing interests}

The authors declare that they have no competing interests.

\section{Funding}

This work was supported by grants from the National Institutes of Health to JES: P01-HL119165.

\section{Authors' contributions}

AHK participated in designing the experiments with JES, executed all experiments, generated and analyzed data, and wrote and revised the manuscript.

JES directed the study and wrote, guided, and revised the manuscript.

PHF generously gave her valuable comments in writing and revising the manuscript.

The authors wish to thank Ana Wang, Ph.D. for assistance in preparing the manuscript.

All authors read and approved the final manuscript.

\section{Acknowledgements}

We thank the National Heart, Lung, and Blood Institute for providing research funding. We also thank the UCSD histology staff for providing histological tissue services in this study.

\section{Author details}

Authors are affiliated with the Proteogenomics Research Institute for Systems Medicine (PRISM), 505 Coast Blvd. South, Suite 209, La Jolla, CA, 92037. 


\section{References}

1.

Ryerson CJ, Kolb M. The increasing mortality of idiopathic pulmonary fibrosis: fact or fallacy? Eur Respir J, 2018. 51(1).

2.

Upagupta C, et al., Matrix abnormalities in pulmonary fibrosis. Eur Respir Rev, 2018. 27(148).

3.

Denayer T, Stöhr T, Van Roy M. Animal models in translational medicine: Validation and prediction. New Horizons in Translational Medicine. 2014;2(1):5-11.

4.

Peng R, et al. Bleomycin induces molecular changes directly relevant to idiopathic pulmonary fibrosis: a model for "active" disease. PLoS One. 2013;8(4):e59348.

5 .

Moeller A, et al. The bleomycin animal model: a useful tool to investigate treatment options for idiopathic pulmonary fibrosis? Int J Biochem Cell Biol. 2008;40(3):362-82.

6.

Zhao H, et al. Pulmonary delivery of docosahexaenoic acid mitigates bleomycin-induced pulmonary fibrosis. BMC Pulm Med. 2014;14:64.

7.

Judge $\mathrm{JL}$, et al. Prevention and treatment of bleomycin-induced pulmonary fibrosis with the lactate dehydrogenase inhibitor gossypol. PLoS One. 2018;13(5):e0197936.

8.

Kulkarni AA, et al. The triterpenoid CDDO-Me inhibits bleomycin-induced lung inflammation and fibrosis.

PLoS One. 2013;8(5):e63798.

9.

Hubner $\mathrm{RH}$, et al. Standardized quantification of pulmonary fibrosis in histological samples.

Biotechniques. 2008;44(4):507-11, 514-7.

10.

Kim SN, et al. Dose-response Effects of Bleomycin on Inflammation and Pulmonary Fibrosis in Mice. Toxicol Res. 2010;26(3):217-22.

11.

Mukherjee S, et al. Disruption of Calcium Signaling in Fibroblasts and Attenuation of Bleomycin-Induced Fibrosis by Nifedipine. Am J Respir Cell Mol Biol. 2015;53(4):450-8.

12.

$\mathrm{Li} \mathrm{H}$, et al. Posttreatment with Protectin DX ameliorates bleomycin-induced pulmonary fibrosis and lung dysfunction in mice. Sci Rep. 2017;7:46754.

13.

Luzina IG, et al. Pharmacological In Vivo Inhibition of S-Nitrosoglutathione Reductase Attenuates Bleomycin-Induced Inflammation and Fibrosis. J Pharmacol Exp Ther. 2015;355(1):13-22. 
14.

Arora A, et al. Amifostine Analog, DRDE-30, Attenuates Bleomycin-Induced Pulmonary Fibrosis in Mice. Front Pharmacol. 2018;9:394.

15.

Swaney JS, et al. A novel, orally active LPA(1) receptor antagonist inhibits lung fibrosis in the mouse bleomycin model. Br J Pharmacol. 2010;160(7):1699-713.

16.

Kobayashi T, et al. Bidirectional role of IL-6 signal in pathogenesis of lung fibrosis. Respir Res. 2015;16:99.

17.

Hamada N, et al. Anti-vascular endothelial growth factor gene therapy attenuates lung injury and fibrosis in mice. J Immunol. 2005;175(2):1224-31.

18.

Izbicki G, et al. Time course of bleomycin-induced lung fibrosis. Int J Exp Pathol. 2002;83(3):111-9. 19.

Howell DCJ, et al. Absence of Proteinase-Activated Receptor-1 Signaling Affords Protection from Bleomycin-Induced Lung Inflammation and Fibrosis. Am J Pathol. 2005;166(5):1353-65. 20.

Fernandez IE, Eickelberg O. The impact of TGF-beta on lung fibrosis: from targeting to biomarkers. Proc Am Thorac Soc. 2012;9(3):111-6.

21.

Kang H. Role of MicroRNAs in TGF-beta Signaling Pathway-Mediated Pulmonary Fibrosis. Int J Mol Sci, 2017. 18(12).

22.

Zhao J, et al. Smad3 deficiency attenuates bleomycin-induced pulmonary fibrosis in mice. Am J Physiol Lung Cell Mol Physiol. 2002;282(3):L585-93.

23.

Willis BC, Borok Z. TGF-beta-induced EMT: mechanisms and implications for fibrotic lung disease. Am J Physiol Lung Cell Mol Physiol. 2007;293(3):L525-34.

24.

Park SW, et al. Interleukin-13 and its receptors in idiopathic interstitial pneumonia: clinical implications for lung function. J Korean Med Sci. 2009;24(4):614-20.

25.

Chung SI, et al. IL-13 is a therapeutic target in radiation lung injury. Sci Rep. 2016;6:39714. 26.

Hashimoto S, et al. IL-4 and IL-13 induce myofibroblastic phenotype of human lung fibroblasts through cJun NH2-terminal kinase-dependent pathway. J Allergy Clin Immunol. 2001;107(6):1001-8.

27.

Zhao J, et al. Sphingosine-1-phosphate receptor-2 facilitates pulmonary fibrosis through potentiating IL13 pathway in macrophages. PLoS One. 2018;13(5):e0197604. 
28.

Saito F, et al. Role of interleukin- 6 in bleomycin-induced lung inflammatory changes in mice. Am J Respir Cell Mol Biol. 2008;38(5):566-71.

29.

Habiel DM, Hogaboam C. Heterogeneity in fibroblast proliferation and survival in idiopathic pulmonary fibrosis. Front Pharmacol. 2014;5:2.

30 .

O'Donoghue RJ, et al. Genetic partitioning of interleukin-6 signalling in mice dissociates Stat3 from Smad3-mediated lung fibrosis. EMBO Mol Med. 2012;4(9):939-51.

31.

Le TT, et al. Blockade of IL-6 Trans signaling attenuates pulmonary fibrosis. J Immunol. 2014;193(7):3755-68.

32.

Klee S, et al. WISP1 mediates IL-6-dependent proliferation in primary human lung fibroblasts. Sci Rep. 2016;6:20547.

33.

Aumiller V, et al. WNT/beta-catenin signaling induces IL-1 beta expression by alveolar epithelial cells in pulmonary fibrosis. Am J Respir Cell Mol Biol. 2013;49(1):96-104.

34.

Henderson WR Jr, et al. Inhibition of Wnt/beta-catenin/CREB binding protein (CBP) signaling reverses pulmonary fibrosis. Proc Natl Acad Sci U S A. 2010;107(32):14309-14.

35 .

Konigshoff $\mathrm{M}$, et al. Functional Wnt signaling is increased in idiopathic pulmonary fibrosis. PLoS One. 2008;3(5):e2142.

36.

Konigshoff $\mathrm{M}$, et al. WNT1-inducible signaling protein-1 mediates pulmonary fibrosis in mice and is upregulated in humans with idiopathic pulmonary fibrosis. J Clin Invest. 2009;119(4):772-87.

37.

Barratt SL, et al., VEGF (Vascular Endothelial Growth Factor) and Fibrotic Lung Disease. Int J Mol Sci, 2018. 19(5).

38.

Madtes DK, et al. Selective induction of tissue inhibitor of metalloproteinase-1 in bleomycin-induced pulmonary fibrosis. Am J Respir Cell Mol Biol. 2001;24(5):599-607.

39.

Bonnans C, Chou J, Werb Z. Remodelling the extracellular matrix in development and disease. Nat Rev Mol Cell Biol. 2014;15(12):786-801.

40.

Manoury B, et al. TIMP-1 is a key factor of fibrogenic response to bleomycin in mouse lung. Int $\mathrm{J}$ Immunopathol Pharmacol. 2006;19(3):471-87.

41. 
Liu X, et al. Does C-reactive protein predict the long-term progression of interstitial lung disease and survival in patients with early systemic sclerosis? Arthritis Care Res (Hoboken). 2013;65(8):1375-80. 42.

Heidari B. The importance of C-reactive protein and other inflammatory markers in patients with chronic obstructive pulmonary disease. Caspian J Intern Med. 2012;3(2):428-35.

\section{Figures}
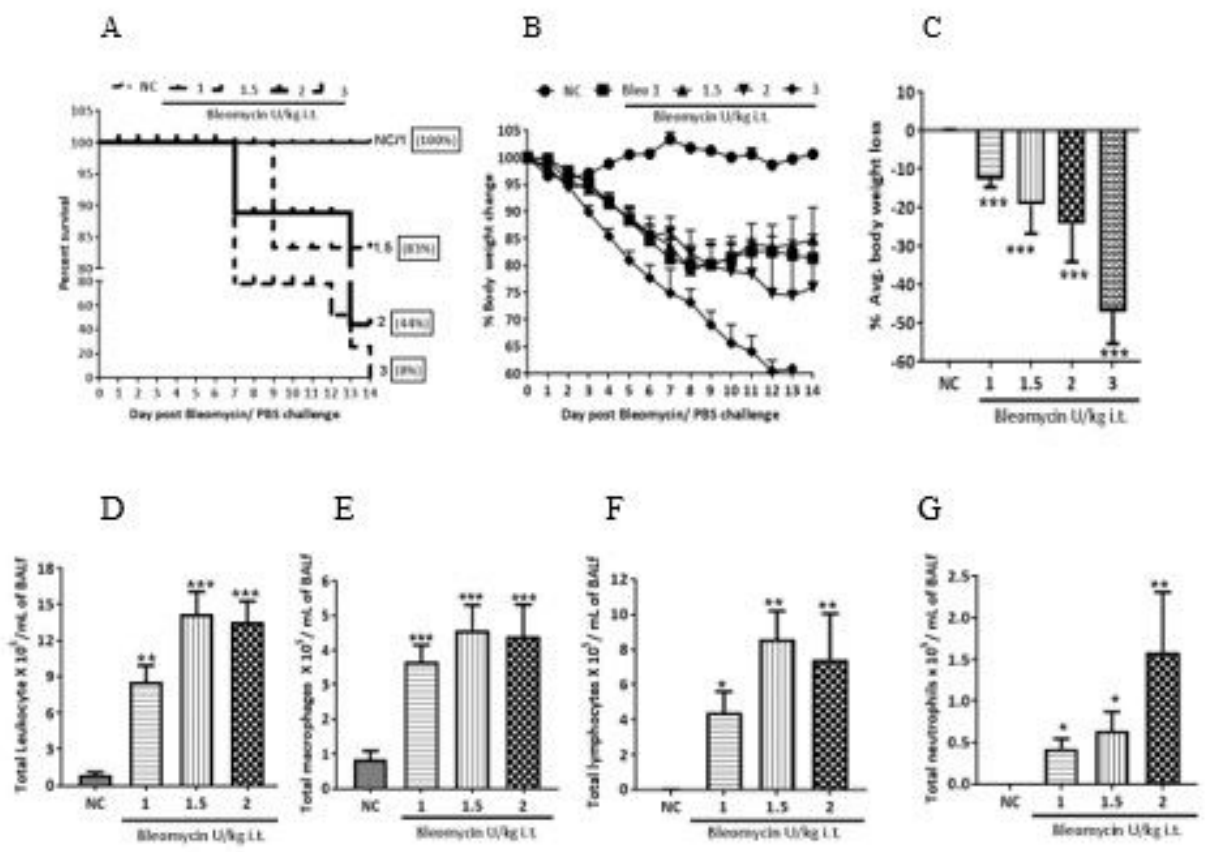

\section{Figure 1}

Effect of intratracheal (IT) high bleomycin doses on clinical signs and pulmonary inflammation: (a) \% survival (Kaplan-Meier plots), (b) \% weight change, and (c) \% avg. weight loss, and levels of (d) leukocytes cells (TLC), (e) macrophages, (f) lymphocytes, and (g) neutrophils. Data are expressed as mean \pm SEM of $n=3-5$ mice/group; ${ }^{*}<<0.05 ;{ }^{* \star} p<0.01$; and ${ }^{* \star *} p<0.001$ vs. control.
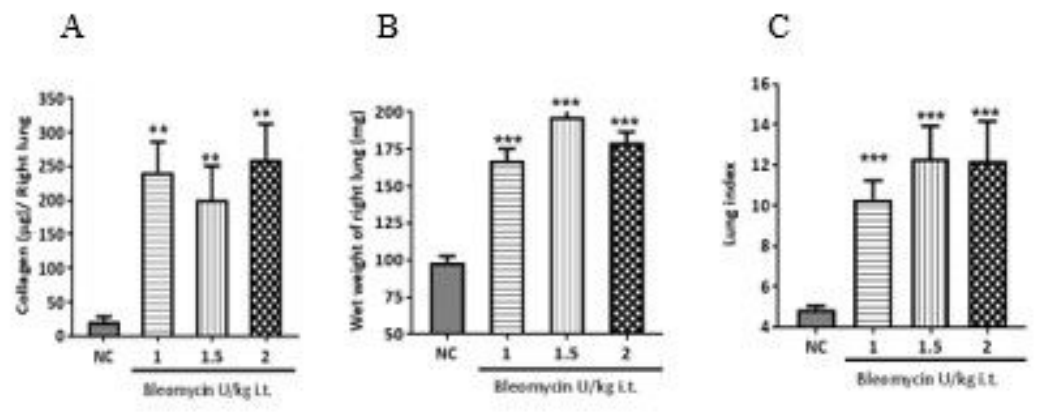
Figure 2

Effect of IT high bleomycin doses on lung parameters (a) right lung collagen content, (b) wet weight of right lung, and (c) lung index. Data are expressed as mean \pm SEM of $n=3-5$ mice/group. ${ }^{*} p<0.05$; $\star * p<0.01 ;$ and $* * * p<0.001$ vs. control.
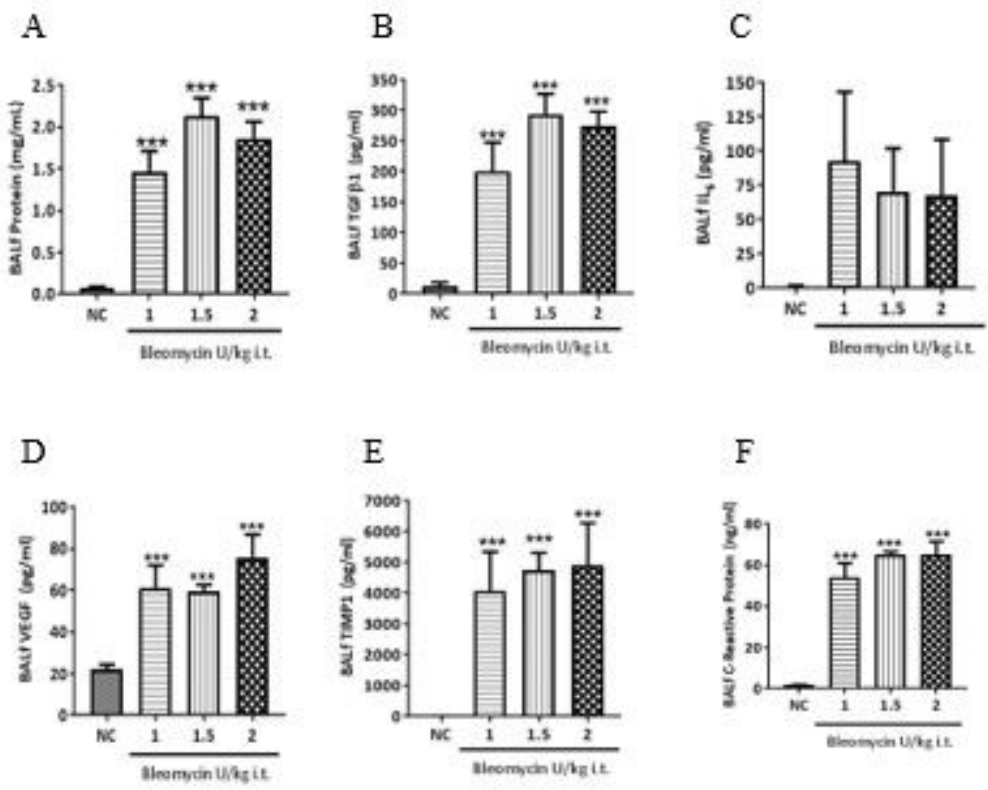

\section{Figure 3}

Effect of IT high bleomycin doses on lung fibrosis biomarkers and vascular leakiness in BALf: (a) Total BALf protein content; profibrotic biomarkers (b) TGF $\beta 1$, (c) IL-6, and (d) VEGF; angiogenesis biomarker (d) VEGF; tissue remodeling biomarker (e)TIMP1; indicator of lung function (f) c-Reactive protein. Data are expressed as mean \pm SEM of $n=3-5$ mice/group. ${ }^{*} p<0.05 ;{ }^{* *} p<0.01$; and ${ }^{* \star *} p<0.001$ vs. control. 
A.

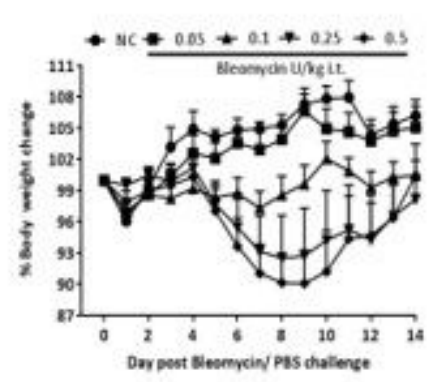

D

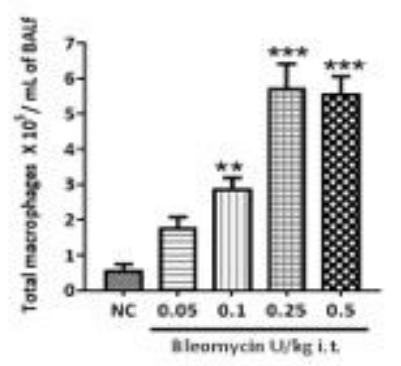

B

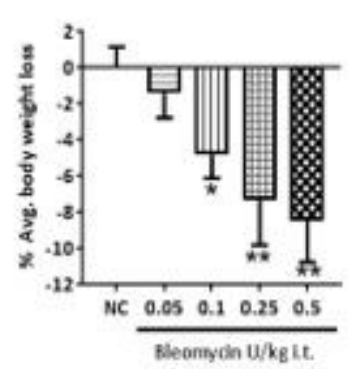

E

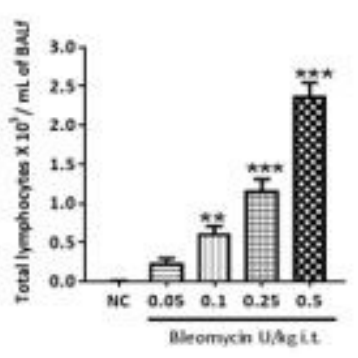

$\mathrm{C}$

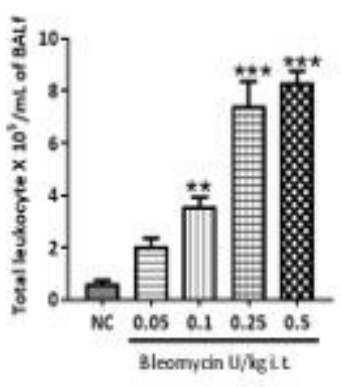

F

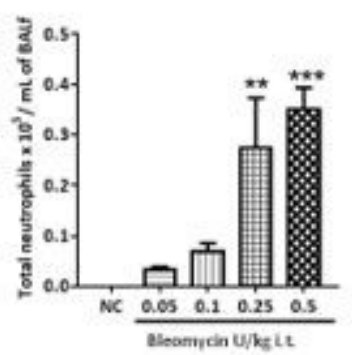

Figure 4

Effect of IT low bleomycin (low doses on clinical signs and pulmonary inflammation: (a) \% weight change, (b) \% avg. weight loss, and levels of (c) leukocytes, (d) macrophages, (e) lymphocytes, and (f) neutrophils. Data are expressed as mean \pm SEM of $n=5$ mice/group. ${ }^{*} p<0.05 ;{ }^{*} p<0.01$; and ${ }^{* \star *} p<0.001$ vs. control.
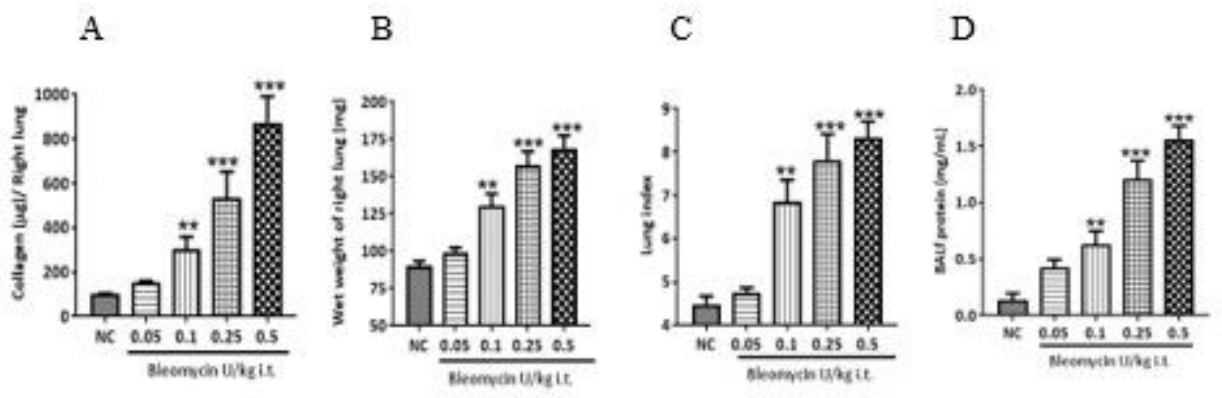

Figure 5

Effect of IT low bleomycin doses on lung parameters and vascular leakiness: (a) Right lung collagen content, (b) wet weight of right lung, (c) lung index, and (d) protein content. Data are expressed as mean \pm SEM of $n=5$ mice/group. ${ }^{*} p<0.05 ;{ }^{* \star} p<0.01$; and ${ }^{* \star *} p<0.001$ vs. control. 
A

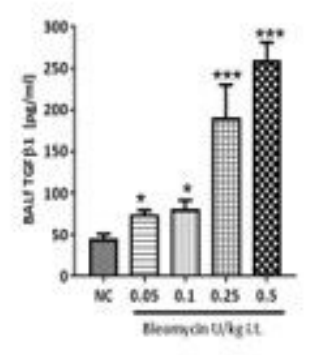

E

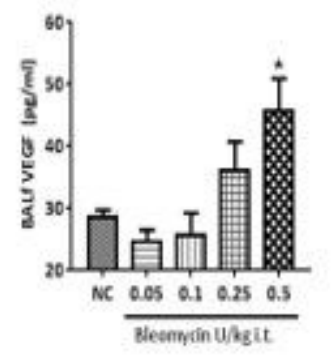

B

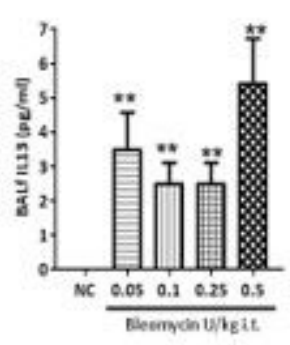

$\mathrm{F}$

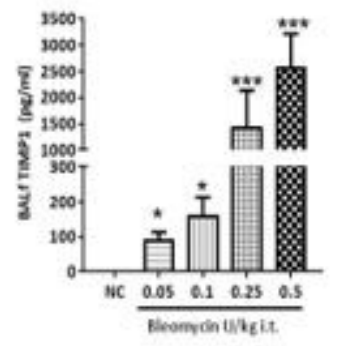

$\mathrm{C}$

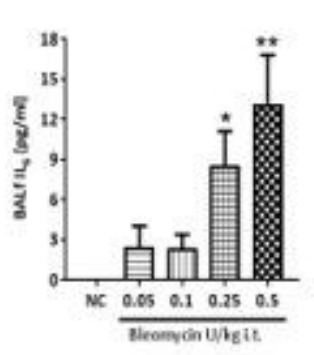

G

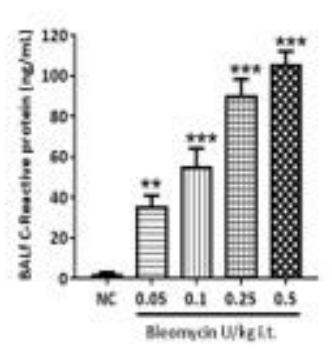

$\mathrm{D}$

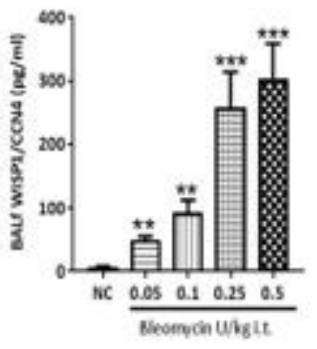

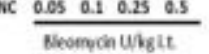

Figure 6

Effect of IT low bleomycin doses) on lung fibrosis biomarkers in BALf :(a) TGFß1, (b) IL-13, (c) IL-6, (d) WISP1, (e)VEGF; angiogenesis biomarker (e)VEGF; tissue remodeling biomarker (f) TIMP1; lung function biomarker (g) c-Reactive protein. Data are expressed as mean \pm SEM of $n=5$ mice/group. ${ }^{*} p<0.05$; ${ }^{* *} p<0.01$; and ${ }^{* * *} \mathrm{p}<0.001$ vs. control.
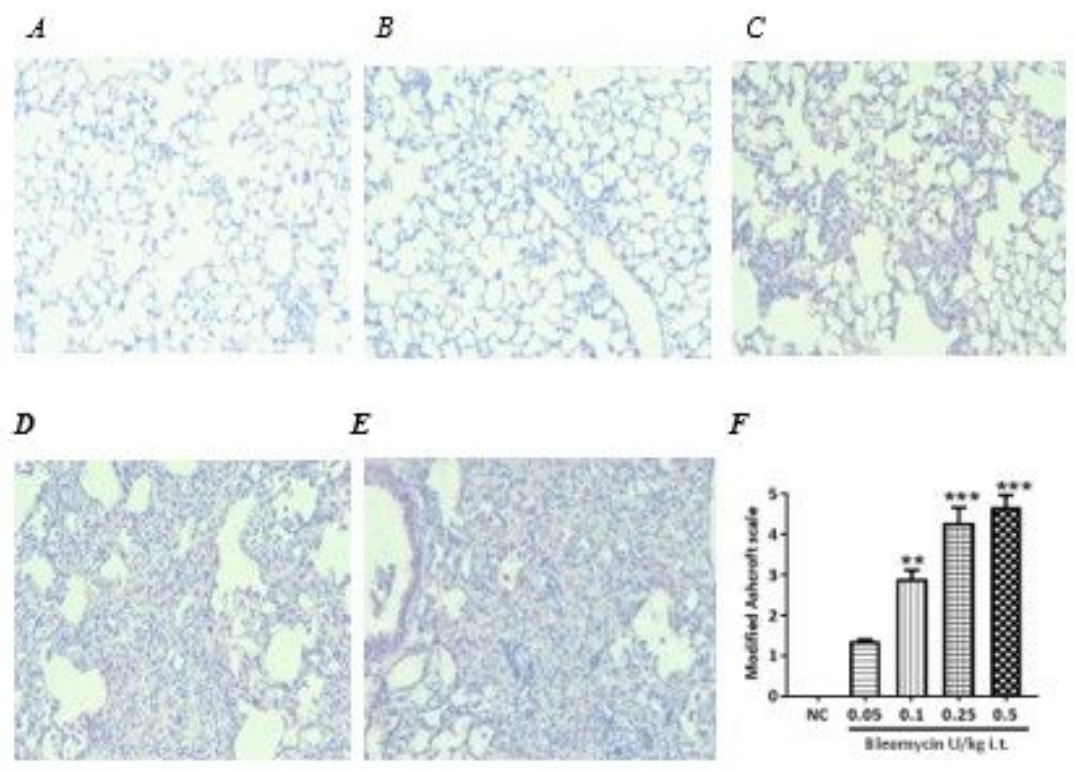

Figure 7 
Effect of IT low bleomycin doses) on lung pathology and severity of fibrosis. Representative histopathological images (100X magnification) of haematoxylin $(\mathrm{H})$ and eosin $(\mathrm{E})$ staining of mice lung treated with (a) PBS, (b) $0.05 \mathrm{U} / \mathrm{kg}$ bleomycin , (c) $0.1 \mathrm{U} / \mathrm{kg}$ bleomycin , (d) $0.25 \mathrm{U} / \mathrm{kg}$ bleomycin, (e) 0.5 $\mathrm{U} / \mathrm{kg}$ bleomycin; severity of fibrosis ( $\mathrm{f}$ ) modified Ashcroft scale. Data are expressed as mean \pm SEM of $\mathrm{n}=5$ mice/group. ${ }^{*} \mathrm{p}<0.05 ;{ }^{* \star} \mathrm{p}<0.01$; and ${ }^{* \star *} \mathrm{p}<0.001$ vs. control.

\section{Supplementary Files}

This is a list of supplementary files associated with this preprint. Click to download.

- CoverletterandSupportingdocuments20200205.docx 